\title{
Parameter identification and Synchronization of different chaotic systems
}

\author{
Yuehua Huang, Yuhuan Jiang ,Lingping Li,Chaoping Rao \\ College of Electrical Engineering \& New Energy, China Three Gorges \\ University, Yichang, 443000,China
}

\begin{abstract}
In order to study the synchronization and parameter identification of different chaotic systems with uncertain parameters, a parameter identifier and a full synchronic controller are designed based on Ulyanovsk stability theory. Take LÜ system as target system and Rossler system as response system to verify its effectiveness. The simulation results show that LÜ system and Rossler system can achieve complete synchronization by the parameter identifier and synchronization controller, and the parameters of LÜ chaos system can all get recognition.

Keywords: full synchronization, parameter identification,stability theory
\end{abstract}

\section{Introduction}

Chaos is a kind of common phenomenon, since Pecora and Carroll proposed the concept of chaotic synchronization and applied it to the circuit for the first time in 1990, the application potential of chaotic synchronization in communication and control in industries had been discovered ${ }^{[1-2]}$. In order to realize the application of chaos in practical, the first important thing to solve is chaos synchronization ${ }^{[3]}$.

There are a lot of methods and techniques to realize chaotic synchronization , such as drive - response method, APD splitting method, the mutual coupling method, adaptive method, variable feedback perturbation method ${ }^{[4-6]}$. Park put forward the adaptive method to the unknown parameters of of uncertain chaotic systems, and realized the synchronization control of super Chen system ${ }^{[7]}$, Kim used the adaptive method for the adaptive synchronization control of Duffing systems $^{[8]}$, Elabbasy completed the adaptive synchronization of uncertain systems $^{[9]}$. The researches above are synchronizations of the same structures and parameters determined. Since the two chaotic systems with the same structures have the same nonlinear function, only the initial conditions of two systems are different, it is relatively easy to achieve the synchronization. Meanwhile, it is almost impossible to find two completely same chaotic systems in the practical application. And due to the reason of the system itself or the limitations of actual application technology, the system parameters are unstable and cannot be accurately predetermined . 
This paper design a parameter identifier and a full synchronic controller based on the theory of stability, which make the two different structure of the chaotic systems to achieve synchronization. When two systems achieve synchronization, the parameters of the response system and the target system achieve consistently, synchronous controller disappear right now, all the parameters of the target system are identified.

\section{Synchronization principles}

Consider the nonlinear dynamic equation of chaotic system as

$$
\begin{aligned}
& \dot{x}=f(x) \quad x \in R^{n} ; \\
& \dot{y}=f(y) \quad y \in R^{m} ;
\end{aligned}
$$

The two dynamic systems have different structures, to achieve the synchronization of this two systems is to realize

$$
\lim _{t \rightarrow \infty}\|x(t)-y(t)\|=0
$$

while $m=n$, consider $\dot{x}=f(x)$ as target system, $\dot{y}=f(y)$ as response system, exert control $A(t)$ on every component of the response system, that is

$$
\dot{y}=f(y)+A(t) \text {; }
$$

Define the error system of every state variable as $e=y-x$,

$$
\dot{e}=f(y)-f(x)+A(t)
$$

Design the controller as

$$
A(t)=B(t)-f(y)+f(x)
$$

While the error system is

$$
\dot{e}=A(t)+B(t) \text {; }
$$

Assume that the parameters of the target system are unknown, when two systems achieve synchronization, the designed parameter recognition system $C(t)$ can be used to realize the recognition of target parameters, $C(t)$ has to meet the lyapunov stability theory, which is to construct a continuous first order partial derivative of positive definite function $V(x, t)$, and make $V^{\prime}(x, t)$ for the positive definite (or negative semi-definite).

\section{Parameter identifier and fully synchronous controller design}

LÜ System was found in 2002 by Lv Jinhu and Chen Guangrong, the system could be regarded as the transition between Lorenz and Chen chaotic attactor, it has three unstable equilibrium point.

Consider LÜ System as target system, While $a=36, b=3, c=20$, LÜ system is in a chaotic state. 


$$
\left\{\begin{array}{l}
\dot{x}_{1}=f_{1}\left(x_{1}, x_{2}, x_{3}\right)=a\left(x_{2}-x_{1}\right) \\
\dot{x}_{2}=f_{2}\left(x_{1}, x_{2}, x_{3}\right)=-x_{1} x_{3}+c x_{2} \\
\dot{x}_{3}=f_{3}\left(x_{1}, x_{2}, x_{3}\right)=x_{1} x_{2}-b x_{3}
\end{array}\right.
$$

Rossler system as a response system, While $a^{\prime}=0.2, b^{\prime}=0.2, c^{\prime}=5.7 \mathrm{e}$, Rossler system is in a chaotic state.

$$
\left\{\begin{array}{l}
\dot{x}_{1}^{\prime}=f_{1}^{\prime}\left(x_{1}^{\prime}, x_{2}^{\prime}, x_{3}^{\prime}\right)=-x_{2}^{\prime}-x_{3}^{\prime} \\
\dot{x}_{2}^{\prime}=f_{2}^{\prime}\left(x_{1}^{\prime}, x_{2}^{\prime}, x_{3}^{\prime}\right)=x_{1}^{\prime}+a^{\prime} x_{2}^{\prime} \\
\dot{x}_{3}^{\prime}=f_{3}^{\prime}\left(x_{1}^{\prime}, x_{2}^{\prime}, x_{3}^{\prime}\right)=b^{\prime}+x_{1}^{\prime} x_{3}^{\prime}-c^{\prime} x_{3}^{\prime}
\end{array}\right.
$$
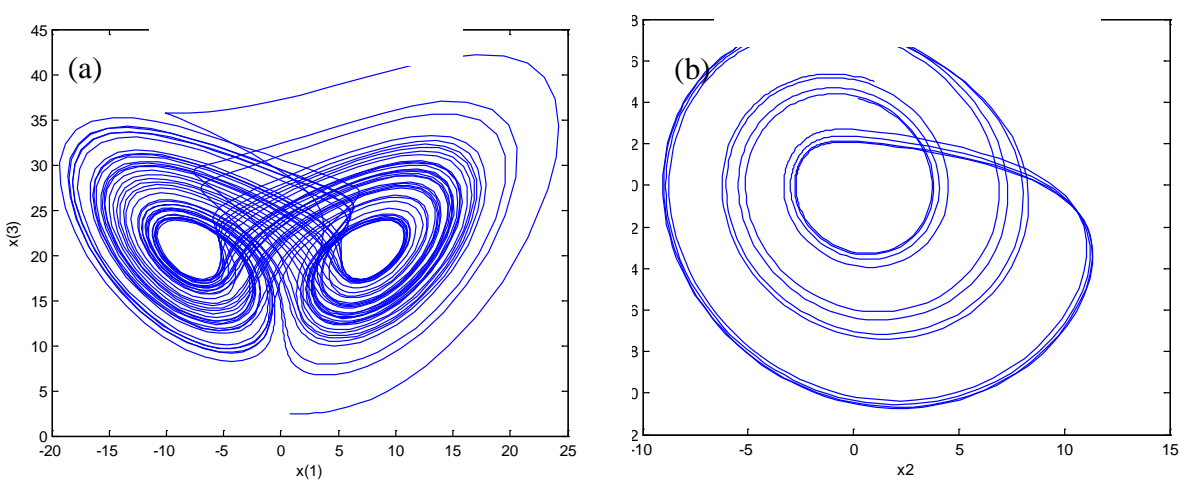

Fig. 1 :(a) LÜ chaos system diagram; (b) Rossler chaos system diagram

Rossler system was found by German scientists Q.Rossler, it has only one non-zero balance point, LÜ system and Rossler system are different chaotic systems both in terms of their nonlinear function forms or graphic structures, so the two systems are chaotic systems of different structures, which meet the conditions for research.

Add the synchronous controllers to the response system as follows:

$$
\left\{\begin{array}{l}
\dot{x}_{1}^{\prime}=f_{1}^{\prime}\left(x_{1}^{\prime}, x_{2}^{\prime}, x_{3}^{\prime}\right)=-x_{2}^{\prime}-x_{3}^{\prime}+u_{1} \\
\dot{x}_{2}^{\prime}=f_{2}^{\prime}\left(x_{1}^{\prime}, x_{2}^{\prime}, x_{3}^{\prime}\right)=x_{1}^{\prime}+a^{\prime} x_{2}^{\prime}+u_{2} \\
\dot{x}_{3}^{\prime}=f_{3}^{\prime}\left(x_{1}^{\prime}, x_{2}^{\prime}, x_{3}^{\prime}\right)=b^{\prime}+x_{1}^{\prime} x_{3}^{\prime}-c^{\prime} x_{3}^{\prime}+u_{3}
\end{array}\right.
$$

Define the error system as

$$
\left\{\begin{array}{l}
e_{1}=x_{1}^{\prime}-x_{1} \\
e_{2}=x_{2}^{\prime}-x_{2} \\
e_{3}=x_{3}^{\prime}-x_{3}
\end{array}\right.
$$


Get the changing process of error variables by time from Formula(1)(2)and(3) , get Formula(5).

$$
\left\{\begin{array}{l}
\dot{e}_{1}=-x_{2}^{\prime}-x_{3}^{\prime}-a\left(x_{2}-x_{1}\right)+u_{1} \\
\dot{e}_{2}=x_{1}^{\prime}+a^{\prime} x_{2}^{\prime}+x_{1} x_{3}-c x_{2}+u_{2} \\
\dot{e}_{3}=b^{\prime}+x_{1}^{\prime} x_{3}^{\prime}-c^{\prime} x_{3}^{\prime}-x_{1} x_{2}+b x_{3}+u_{3}
\end{array}\right.
$$

Assume that all the parameters of the system (1) are unknown, to make the goal system(1) and response system (2) completely realize synchronization, synchronization controller is designed as $u=\left(u_{1}, u_{2}, u_{3}\right)$;

$$
\left\{\begin{array}{l}
u_{1}=\bar{a}\left(x_{2}-x_{1}\right)-28 e_{1}+e_{2}+e_{3}+x_{2}+x_{3} \\
u_{2}=-15 e_{2}-e_{1}-x_{1}-a^{\prime}\left(e_{2}+x_{2}\right)-x_{1} x_{3}+\bar{c} x_{2} \\
u_{3}=-2 e_{3}-b^{\prime}-\left(e_{1}+x_{1}\right)\left(e_{3}+x_{3}\right)+c^{\prime}\left(e_{3}+x_{3}\right)+x_{1} x_{2}-\bar{b} x_{3}
\end{array}\right.
$$

Parameters $\bar{a}, \bar{b}, \bar{c}$ are estimated values of $a, b, c$.

Define the error variable $e_{a}=\bar{a}-a, e_{b}=\bar{b}-b, e_{c}=\bar{c}-c$.

Design parameter identifier as

$$
\left\{\begin{array}{l}
\dot{\bar{a}}=-\left(x_{2}-x_{1}\right) e_{1} \\
\dot{\bar{b}}=x_{3} e_{3} \\
\dot{\bar{c}}=-x_{2} e_{2}
\end{array}\right.
$$

Theorem For response system (2), if the synchronous controller satisfy Formula(6), the parameter identifier satisfy Formula(7), the target system (1) and the response system (2) can be synchronized for any initial values.

Proof Design Lyapunov function

$$
V(t)=\frac{1}{2}\left(e_{1}^{2}+e_{2}^{2}+e_{3}^{2}+e_{a}^{2}+e_{b}^{2}+e_{c}^{2}\right)
$$

Get derivative of the Formula $V(t)$

$$
\begin{aligned}
\dot{V}(t) & =\dot{e}_{1} e_{1}+\dot{e}_{2} e_{2}+\dot{e}_{3} e 3+\dot{e}_{a} e_{a}+\dot{e}_{b} e_{b}+\dot{e}_{c} e_{c} ; \\
& =-28 e_{1}^{2}-15 e_{2}^{2}-2 e_{3}^{2} \leq 0
\end{aligned}
$$

$\dot{V}(t)$ is negative semidefinite, the system is consistent and stable by Lyapunov stability theory. When $\dot{V}(t) \leq 0$, there is $e_{1}, e_{2}, e_{3} \in L_{\infty}, e_{a}, e_{b}, e_{c} \in L_{\infty}, \dot{e}_{1}, \dot{e}_{2}, \dot{e}_{3} \in L_{\infty}$, as $\dot{V}=-e^{T} e$,

$$
\int_{0}^{t}\|e\|^{2} d t=\int_{0}^{t} e^{T} e d t=-\int_{0}^{t} \dot{V} d t=V(0)-V(t) \geq 0
$$


In whcin $V(0)$ is the initial value of Formula (11), $V(0)$ is bounded, so $e_{1}, e_{2}, e_{3} \in L_{2}$, according to Barbalat Lemma, whiel $t \rightarrow 0$, $e_{1}, e_{2}, e_{3} \rightarrow 0, \lim _{t \rightarrow \infty}\|e(t)\|=0$, the error is tend to zero, that means target system and response system gradually achieve synchronization.

\section{Simulation}

Simulation experiment using MATLAB in synchronization, take the initial value of the target system and response system respectively $x_{1}(0)=-1, x_{2}(0)=0.4$, $x_{1}^{\prime}(0)=0.3, x_{2}^{\prime}(0)=0.2, x_{3}^{\prime}(0)=0.1 x_{3}(0)=0.1$, take estimated parameters of the initial value $\bar{a}(0)=0.8, \bar{b}(0)=0, \bar{c}(0)=1$.

Fig. 2 .(a)shows the changes of state variable $x(1)$ and $x(1)^{\prime}$ in two systems by time. Fig. 2.(b)shows the error variables by time, the state variable error are close to zero by time, that is when the two systems to achieve complete synchronization. Fig. 5.shows the parameter identification process. The unknown parameters gradually tend to be $36,3,20$ in the $40 \mathrm{~s}, 5 \mathrm{~s}, 2 \mathrm{~s}$, which achieved the goal of parameter identification, it suggests that parameter identifier is effective.
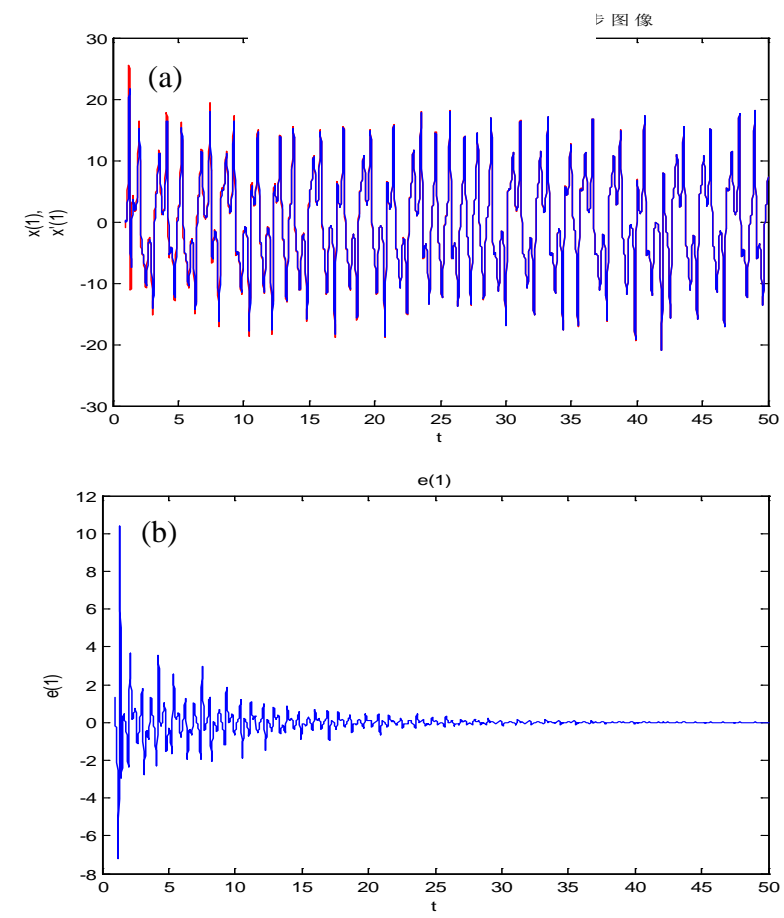

Fig. 2: (a) The changes of state variable $x(1)$ and $x(1)$ ' by time;(b)The changes of $x(1)-x(1)$ 'by time 

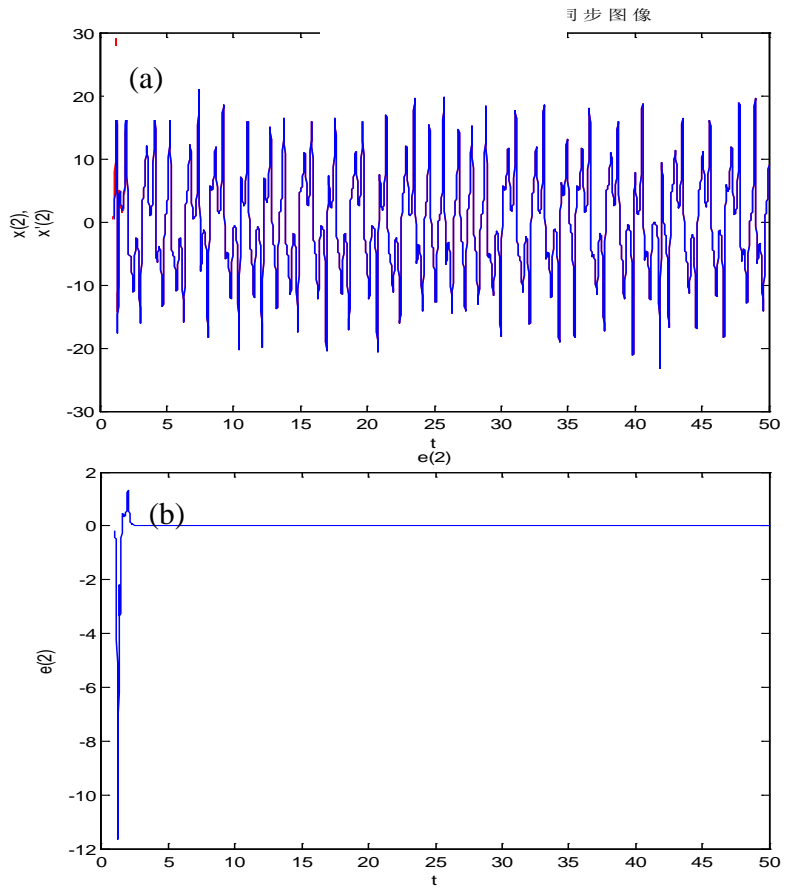

Fig. 3: (a) The changes of state variable $\mathrm{x}(2)$ and $\mathrm{x}(2)$ ' by time;(b)The changes of $x(2)$-x(2)'by time

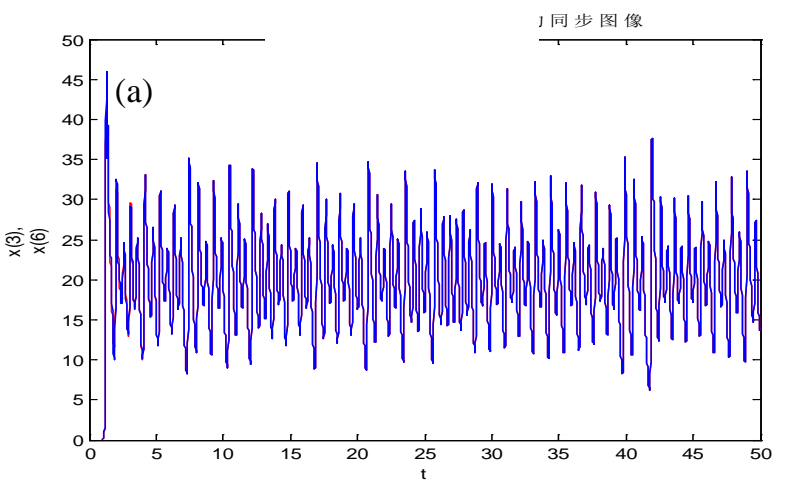




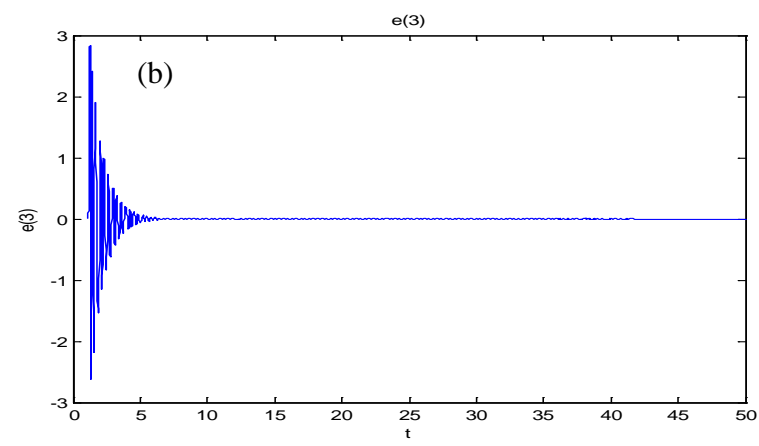

Fig.4: (a) The changes of state variable $\mathrm{x}(3)$ and $\mathrm{x}(3)$ ' by time;(b)The changes of $x(3)-x(3)$ 'by time

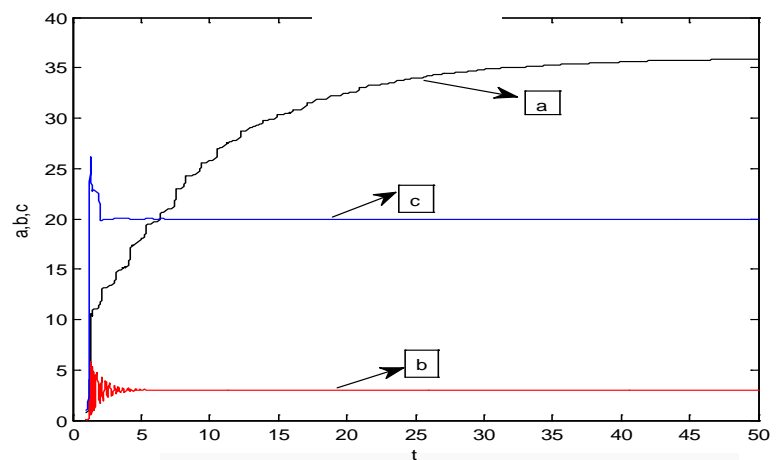

Fig. 5: parameter identification process

\section{Conclusion}

Based on the theory of stability, a parameter identifier and full synchronic controller are designed, analalyzed the feasibility of theidentifier and the controller by Ulyanovsk stability theory. Simulation test furtherly verifed the effectiveness of the control scheme, two different chaos systems can realize synchronization and all parameters can be identified by the parameter identifier and full synchronic controller.

\section{Acknowledgements}

The research work was supported by National Natural Science Foundation of China under Grant No. 51177088.

\section{References}


[1] H.N.Agiza.Chaos synchronization of Lu dynamical system[J],Nonlinear Analysis,2004,58:10-11

[2] WANG Qi. Bidirectional Partial Generalized Synchronization in Chaotic and Hyperchaotic Systems via a New Scheme[J],ommun.Theor.hys. 2006,45(6):1049-1056

[3] KMYG.KMJ KOU CC,etc. TCP-friendly internet video with smooth and fast rate adaptation and network-aware erro cotrol[J]. IEEE Trans on Circuits and Systems for Video Technology, 2004,14(2):256-268.

[4] PAL T,PAL N R,PAL M. Learning fuzzy rules for controllers with genetic algorithms[J]. International Journal of Intelligent Systems,2003,18(5):569-592

[5] Lü J, Chen G. A new chaotic attractor coined [J]. Int. J. Bifur. Chaos2002, 12(3): 659-66

[6] Liu C X, Liu T, Liu L, et al. A new chaotic attractor [J]. Chaos, Solitons and Fractals, 2004, 22:1031-038.

[7] Kim J H, Park C W, Kim E, Park M 2005Phys. Lett. A334295

[8] Elabbasy EM, Agiza H N, EI-Dessoky M M 2004Chaos, Solitons and Fractals21657

[9] CHEN Jun, LIU Zenrong. Partial synchroniztion between different systems[J]Applied Mathematics and Mechanics,2005,26(9):1232-1136 